\title{
DOS PARIENTES DENTRO DE LA FAMILIA SEMANTICISTA EN LA FILOSOFÍA DE LA CIENCIA ${ }^{1}$
}

\author{
Leonardo Cárdenas Castañeda* \\ leonardo.cardenas@ucaldas.edu.co
}

RESUMO Este artigo se inicia com a declaração de Díez e Moulines, segundo a qual é preferível dizer 'família semanticista' e não 'concepção semanticista', desde que, entretanto, os membros dessa família partilhem uma estratégia modal de apresentação e análise das teorias e, claro, de problemas metafóricos ligados a eles, ainda, entre eles não deve haver qualquer unanimidade em relação à natureza dos modelos. O estruturalismo, por exemplo, pensa os modelos como estruturas de conjunto teórico (ordenada por múltiplo), enquanto para van Fraassen elas são 'pontos'ou 'trajetórias em fase de espaço'. Assim, o objetivo do presente trabalho consiste em pesquisar como é considerada a noção de modelo nesses dois membros da família semanticista, a despeito de sua relação em sua formulação inicial.

Palavras-chave Família semanticista, estruturalismo, empirismo construtivo, modelo teórico.

ABSTRACT This article begins with the statement of Diez and Moulines, in which it is preferable to say 'semanticist family' and not

1 Este artículo es un avance de mi proyecto de investigación doctoral titulado: "En Defensa de una Epistemología Naturalista Alternativa: Respuesta a un Falso Dilema", llevado a cabo en la Universidad Pontificia Bolivariana (Medellín). Agradezco al evaluador anónimo por sus amables sugerencias y comentarios críticos sobre este trabajo.

* Profesor del Departamento de Filosofía de la Universidad de Caldas (Manizales - Colombia). Artigo recebido em 13/07/2013 e aprobado em 20/11/2013. 
'semanticist conception', since, even though, the members of this family share a modal strategy of presentation and analysis of the theories and, of course, of metatheoric problems linked to them, also, between them there is no unanimity in relation to the nature of the models. Structuralism, for example, thinks in the models as structures of theory set (n-tuple ordered), while for van Fraassen are 'points' or 'trajectories in space phase'. Therefore, the aim of this paper consists to research how in these two members of semanticist family is assumed the notion of model, despite its relationship in its initial formulation.

Keywords Family semanticist, structuralism, constructive empiricism, theoretical model.

\section{Introducción}

El período del cual me ocuparé en el presente artículo corresponde a lo que se ha denominado concepción semanticista de las teorías, en la medida en que la semántica va a identificarse con la teoría, no de las proposiciones (a la manera clásica), sino con la de los modelos que intentan representar ciertos objetos. ${ }^{2}$ De allí que este período, nacido finalizando la década del setenta del siglo pasado, se le conozca también con el rótulo de concepción modelística de las teorías. La razón de ello es que, a diferencia de lo que se formuló en la concepción heredada o estándar, las teorías científicas quedan mejor comprendidas como un conjunto de modelos, más que como un conjunto de enunciados, pues dentro de este período las teorías científicas relacionan términos teóricos con términos observacionales mediante reglas de correspondencia, o sea, debe existir una relación isomórfica entre la teoría y el mundo.

Precisamente, parte de las razones que justifican a la concepción semántica o modelística de las teorías se debe a las dificultades que existen al interior de la concepción heredada y que trataré de exponer en el presente trabajo. Ahora, autores como Díez y Moulines (1999), sostienen que es preferible hablar de la "familia semanticista" y no de la "concepción semanticista", pues, como

2 Si bien más adelante mostraré la diferencia entre la concepción heredada con la concepción semanticista, es importante señalar de entrada que en contraste con la primera, en el proyecto semántico o modelístico las teorías no son entidades lingüísticas. Como vamos a ver esto trae como consecuencia de que en la tradición modelo-teórica se hable más de "similitud" o "aproximación empírica", más que de "verdad" o "relaciones ismórficas". 
ellos aclaran, aunque los miembros de esta familia comparten la estrategia modelística de presentación y análisis de las teorías y, por supuesto, de los problemas metateóricos a ellas ligados, entre ellos no existe unanimidad con relación a la naturaleza de los modelos. El estructuralismo, por ejemplo, los concibe como estructuras conjuntistas (n-tuplos ordenados) y van Fraassen (1996) como puntos o trayectorias en un espacio de fases. Así pues, el objetivo de este artículo consiste en rastrear la manera como dentro de la familia semanticista se asume la noción de modelo, a pesar de sus parentescos en su formulación inicial.

\section{Críticas a la concepcion heredada}

Si bien las críticas realizadas por los filósofos historicistas a la concepción heredada fueron importantes, en particular con la llamada "carga teórica de la observación", los partidarios de la familia semanticista tienen razones independientes de peso para dudar del enfoque sintacticista adoptado por la concepción estándar.

Una primera objeción consiste en que resulta muy complicado en la práctica tratar de reducir todas las teorías a la lógica de predicados de primer orden. Es decir, la labor de examinar la identidad de una teoría a través de cálculos axiomáticos y de reglas de correspondencia expresado en un lenguaje formal no ofrece ninguna utilidad práctica. Más aun cuando gran parte de las teorías científicas están compuestas por un lenguaje matemático especializado que no necesariamente es susceptible de ser interpretado mediante la lógica de predicados de primer orden, o por lo menos dicha tarea resultaría altamente engorrosa. ${ }^{3}$

En segundo lugar, y siguiendo con la idea anterior, la propuesta formalista de la concepción heredada sigue siendo poco práctica por el hecho de que muchas de las teorías científicas están conectadas con otras teorías, razón por la cual tendríamos que vernos tentados a traducir al puro cálculo de predicados todo ese conjunto de teorías previas si queremos que nuestro análisis metateórico tenga éxito. Sin embargo tal análisis constituye un regreso ad infinitum. Pensemos solamente en todas las teorías que deberíamos reducir a cálculos formales al estilo de Hilbert a la Mecánica Clásica de Partículas para

3 Me parece relevante mencionar en esta parte del artículo que representantes de la concepción heredada desarrollaron algunos proyectos posteriores de utilizar lógicas modales y de orden superior, donde toman distancia de la lógica de predicados de primer orden. El capítulo V del libro de Rudolf Carnap (1956) "Meaning and Necessity" en el que examina la naturaleza de las modalidades es un buen ejemplo de ello. 
su análisis, pues dentro de esta teoría física existen otras de las cuales dispone, como teorías propias de la geometría, de la mereología o de la cronometría.

Una tercera crítica a la concepción clásica de las teorías consiste en mostrar la inconsistencia de la definición inicial de lo que en esta tradición se entiende por teoría. Como lo menciona Díez y Moulines, si nos tomamos en serio el asunto de que las teorías son un conjunto de enunciados, entonces esto tiene la implicación de que al tener dos formulaciones distintas de una misma teoría, debemos suponer que estamos ante dos teorías distintas. Sin embargo esto es un error, pues efectivamente es posible hacer formulaciones distintas de una teoría sin que ésta se altere. Ahora, si esto es así, entonces es falso que las teorías sean un conjunto de enunciados. Díez y Moulines lo ponen en estos términos:

Para apreciar el elemento insatisfactorio más notorio de la concepción sintácticoaxiomática, es imprescindible tomársela en serio, tomarse en serio la identificación de una teoría con una serie de enunciados [...] Según esta concepción, una teoría es una clase de axiomas, y si nos tomamos en serio ello implica que toda diferencia en axiomas supone una diferencia de teorías. Puesto que dos axiomatizaciones diferentes son dos diferentes clases de enunciados, tenemos dos teorías diferentes. Ésta es una consecuencia intuitivamente insatisfactoria, pues podemos tener dos axiomatizaciones diferentes de, intuitivamente, "la misma teoría" [...] Parece intuitivamente razonable que pueda haber axiomatizaciones diferentes de una misma teoría, esto es, que no hay ningún sentido interesante en que quepa hablar de dos teorías. Si eso es así, una teoría no puede ser un conjunto de axiomas, no se representa metateóricamente de forma satisfactoria identificándola con un conjunto tal. (Diez; Moulines, 1999, pp. 328-329)

Por último, quisiera desarrollar otra réplica que viene de una de las familias semanticistas, se trata de la discusión en filosofía de la ciencia sobre el problema de la teoricidad que autores estructuralistas como Joseph Sneed (1971) y el mismo Carlos U. Moulines (1982) han propuesto para elaborar una opción alternativa a la de la concepción heredada. Recordemos que para la visión clásica de las teorías el lenguaje de la ciencia se divide entre términos teóricos (aquellos que no poseen una manera directa de observación empírica tales como el electrón, molécula, el átomo) y términos observacionales o no teóricos (aquellos que tienen una referencia directa con la experiencia como el color y la dureza) que se relacionan a través de reglas de correspondencia o de interpretación, pues la idea es reducir los términos teóricos a lo puramente observacional. De ahí que también se reconozca a los filósofos de la concepción heredada, como Carl Hempel (1980), el de asumir el "modelo" de los dos niveles del lenguaje. 
El punto es que el compromiso con esta noción de teoría tiene el siguiente yerro, pues conlleva a un error categorial. Una cosa es el átomo como término teórico o que depende o hace parte de una teoría como concepto, y otra el átomo real que podemos experimentar a partir de instrumentos empíricos. Expresado de otra manera, los términos y los conceptos por definición son teóricos; mientras los entes y los objetos por definición son observables. Dice Bas van Fraassen:

Los términos o los conceptos son teóricos (introducidos o adaptados para los propósitos de la construcción de teorías); los entes [v.gr., los átomos] son observables o inobservables. Esto puede parecer un detalle pequeño, pero separa la discusión en dos preguntas: ¿Podemos dividir nuestro lenguaje en una parte teórica y una no teórica? Y, por otro lado, ¿podemos clasificar objetos y eventos en observables e inobservables? (van Fraassen, 1996, p. 30. Corchetes añadidos)

Teniendo en cuenta este tipo de inconvenientes, la concepción estructuralista de las teorías trata de solucionar el problema de la teoricidad con la distinción, no entre el lenguaje teórico y el lenguaje observacional, sino entre términos teóricos y términos no teóricos. Según Moulines, dentro de una teoría debemos identificar los $T$-teóricos como los $T$-no-teóricos. Los primeros son aquellos términos de las teorías que son propios de ella, mientras los segundos son algo así como, términos de teorías subsidiarias o anexas. El mismo Moulines lo expresa de esta manera:

[...] hay que distinguir dos niveles conceptuales y metodológicos dentro de una teoría $T$ dada: el nivel de los conceptos que son específicos de esa teoría, y a los que llamamos conceptos " $T$-teóricos", y el nivel de los conceptos que provienen, por así decir, del "medio ambiente" de $T$, a los cuales llamamos conceptos " $T$-no-teóricos". (Moulines, 2002, p. 17. Corchetes añadidos)

Uno de los ejemplos más comunes a los que apela Moulines para ilustrar esta distinción es la Mecánica Clásica de Partículas, en adelante MCP. En esta teoría física los elementos teóricos básicos son: posición, tiempo y espacio, masa y velocidad. Bien, ahora haciendo caso de la sugerencia del estructuralismo, pasemos a discriminar los $T$-teóricos y los $T$-no-teóricos de la MCP.

Son propias de esta teoría física la masa y la velocidad. Es decir, estos conceptos son genuinos o pertenecen al nivel de la física, no requieren de otras teorías auxiliares para poder hacer consistente a la MCP. Así pues, estos conceptos, dada su naturaleza, son $T$-teóricos, "son específicos de esa teoría".

Los términos restantes: la posición, el tiempo y el espacio pertenecen a teorías distintas de la MCP, son términos que funcionan como vínculos 
interteóricos entre teorías distintas. Por ejemplo, el término de espacio no es un término originario de la física sino que pertenece a otra teoría distinta, a la geometría. Lo mismo pasa con la posición y el tiempo, la primera corresponde a la mereología y la segunda a la cronometría. En síntesis, espacio, tiempo y posición son $T$-no teóricos de la MCP; o más bien, la MCP presupone términos que pertenecen a la geometría, a la mereología y a la cronometría. ${ }^{4}$

Naturalmente, una pregunta inmediata que podría hacerse es ¿en qué consiste, entonces, la importancia de esta taxonomía? Nuestra respuesta tendrá dos partes. En primer lugar, la distinción entre términos $T$-teóricos y $T$-no-teóricos muestra por qué la distinción es funcional o pragmática. Es decir, esta distinción no es de carácter universal sino que es relativa a distintas teorías científicas, aquí solo se muestra la importancia para la MCP, pero otras teorías podrían echar mano de esta taxonomía. Solo por mencionar otro ejemplo, en breve, la teoría cinética de los gases posee dentro de los $T$ - teóricos la presión, la temperatura y el volumen; y dentro de los $T$-no-teóricos están la fuerza y la masa. Entonces, los $T$-teóricos, en este caso, hacen parte de la química, mientras que los $T$-no-teóricos, como términos subsidiarios de la teoría cinética de los gases hacen parte de teorías previamente disponibles, en especial de la física. ${ }^{5}$

En segundo lugar, el alcance de la taxonomía consiste en que esta da cuenta no solo de las descripciones, sino que también ofrece explicaciones y predicciones al interior de una teoría. Sigamos con la MCP, cuando queremos saber el valor del movimiento de una partícula (o un punto de masa) debemos averiguar, en primera instancia, el valor de la fuerza con que ese movimiento fue causado, pero también debemos acudir al espacio en donde el suceso se llevó a cabo y al tiempo $i$ inicial y al tiempo $i$, donde se detiene o pierde velocidad la partícula.

Bien, podemos deducir lo siguiente con este elemental ejemplo:

a) Con los $T$-teóricos solo obtendríamos descripciones del evento. $\mathrm{O}$ sea, con la masa y la velocidad se consiguen descripciones o relatos de lo ocurrido. Los juicios serían de este corte: "este cuerpo se mueve a $x$ velocidad", "este cuerpo posee $x$ masa", etc.

4 Hempel (1980, pp. 110-113) Ilamaba a esta relación interteórica como Principio Puente, donde en este caso, la geometría, la cronometría y la mereología son teorías previamente disponibles de la MCP.

5 Debo reconocer que los términos como presión, temperatura y volumen también se asocian con la física. Sin embargo, lo que pretendo mostrar es que la masa y la fuerza sirven como términos anexos para que la teoría cinética de los gases funcione de manera consistente. Por eso, especialmente en este caso, los términos de la masa y fuerza son tomados como $T$-no-teóricos. 
b) Con los $T$-no-teóricos podemos realizar explicaciones de este tipo: "este cuerpo se mueve a $x$ velocidad, dependiendo de las condiciones del espacio donde ocurre el suceso", "la aceleración de este cuerpo varía con respecto al tiempo $i$ y al tiempo $i i "$.

c) Con los $T$-no-teóricos podemos realizar predicciones como sigue: "dependiendo de las condiciones del espacio, probablemente la velocidad de $x$ cuerpo será de $y$ manera", "la aceleración de x cuerpo será de $y$ manera en el tiempo $i$ con respecto al tiempo $i i$, dependiendo de la fuerza con que se impregne".

Dadas estas aclaraciones no entendamos por esto que una teoría que tiene la capacidad de explicar tiene también la capacidad de hacer predicciones; y tampoco a la inversa, una teoría que tiene la capacidad de hacer predicciones tiene la capacidad de realizar explicaciones.

\section{La noción de modelo según la corriente semántica}

De acuerdo a las anteriores réplicas que desde el semanticismo se le han hecho a la concepción heredada, resulta insatisfactorio identificar a las teorías como un conjunto de enunciados sintácticamente interpretados y sus consecuencias. La idea ahora, es identificar a la naturaleza de las teorías como un conjunto de modelos. Sin embargo, esta manera escueta de mencionar tal característica común a toda la "familia semanticista" requiere ciertas precisiones, pues según el uso que se haga de la noción de "modelo", esta puede tener connotaciones distintas.

Por un lado, "modelo" puede significar un objeto que sirve para tratar de figurar o de representar algo, pensemos por ejemplo en un mapa que tiene como función el de tratar de representar a una ciudad; o un plano que pretende figurar la estructura de un edificio. En este caso, tanto el mapa como el plano son modelos de la ciudad y del edificio respectivamente. Por otro lado, el término "modelo" puede significar lo contrario, es decir, referirse con el término no al retrato como tal sino más bien al objeto retratado, por ejemplo un paisaje que sirve como modelo para pintar un óleo.

Obviamente, esta es una manera bastante pictórica y superficial de presentar el asunto, pero lo que se puede intuir con la noción de modelo que adopta la familia semanticista es que la caracterización sintacticista si no es irrelevante, por lo menos es relegada a un segundo plano. van Fraassen lo expresa de este modo: 
De acuerdo con la concepción semántica, presentar una teoría es presentar una familia de modelos. Esta familia puede ser descrita de varios modos, mediante enunciados diferentes en lenguajes diferentes, y ninguna formulación lingüística tiene ningún estatuto privilegiado. Específicamente, no se atribuye ninguna importancia a la axiomatización como tal, e incluso la teoría puede no ser axiomatizable en ningún sentido no trivial. (van Fraassen, 1989, p. 188)

Otro de los aspectos más relevantes dentro de la corriente semántica y su uso de la noción de modelo tiene que ver con la introducción de la formalización lógico - matemática de un predicado conjuntista al estilo de Tarski (1956). Por lo menos, para la versión modelística que asume van Fraassen cuando concibe a las teorías científicas como puntos o trayectorias en un espacio de estados y para los estructuralistas metateóricos que tienen por objetivo la reconstrucción modelo teórica de las teorías. La formulación tarskiana de un predicado conjuntista para la familia semanticista se justifica entonces en este sentido: "Dar un modelo para una teoría es dar una interpretación de sus términos (i.e., dotarlos de un significado concreto) tal que los axiomas (y teoremas) de la misma resulten verdaderos en esa interpretación" (Casanueva, 2005, p. 38).

\section{El estructuralismo y su noción de modelo}

Dentro de la concepción semántica Patrick Suppes (1988) fue quien más aprovechó la anterior idea tarskiana (y que tanto gustó a los miembros del programa estructuralista) para formular su noción de modelo como sistemas abstractos, en el que dado un predicado conjuntista se toma como vocabulario propio de una determinada teoría aquellos términos que no están todavía interpretados o que todavía carecen de contenido empírico. Siendo así, entonces tendríamos por ejemplo la forma del siguiente predicado conjuntista: "x es un modelo de la teoría... $\operatorname{syss}^{6} \operatorname{def}^{7} \varphi(\mathrm{x})$ ", donde $\varphi(\mathrm{x})$ denota las leyes de la teoría y todas sus posibles realizaciones, i.e., sus entidades, relaciones y funciones.

De esta manera, Suppes identifica la parte formal de una teoría y que los estructuralistas como Balzer, Moulines y Sneed en "An Architectonic for Science" (1987) van a llamar el núcleo teórico (K). Claro que la labor la completa Ernest Adams (1959) cuando también identifica la parte aplicativa de una teoría, que es en últimas la interpretación empírica, pues quedarnos solamente con la propuesta de Suppes es tener no más que la formalización

7 Me estoy refiriendo a la definición (def) de los términos que componen la mecánica newtoniana. 
abstracta. El estructuralismo llama a este aporte de Adams como las aplicaciones intencionales o propuestas (I). Por tales razones, la definición de una teoría será $\mathrm{T}=\langle\mathrm{K}, \mathrm{I}\rangle$. Lo que haré entonces es definir las piezas conceptuales que hacen parte tanto del núcleo de la teoría $(\mathrm{K})$ como de las aplicaciones intencionales (I).

\section{El núcleo teórico}

Como ya se mencionó, el núcleo teórico tiene como objetivo el de mostrar las leyes o la parte formal de una teoría, naturalmente, leyes expresadas a partir de modelos, no de enunciados, bajo el proyecto de Suppes. En otros términos, en el núcleo teórico $\mathrm{K}$ se hallan los modelos que cumplen con los axiomas que son propios de una teoría. En el núcleo teórico debemos señalar los modelos potenciales y modelos actuales; las condiciones de ligadura y la noción de T- teoricidad junto con los modelos parciales.

\section{Modelos potenciales y modelos actuales}

En la concepción estructuralista es necesario distinguir los axiomas impropios de una teoría (que definen un tipo de estructura, sin restricciones) y los axiomas propios (el que expresa la ley y ejerce restricciones). Dentro del estructuralismo esto es importante porque se pueden identificar dos clases de modelos, aquellos definidos exclusivamente por los axiomas que llamará Modelos Potenciales (Mp) y aquellos que satisfacen también los axiomas propios (esto es, que cumplen con las leyes) y son llamados Modelos Actuales. En otras palabras, los modelos potenciales (Mp) es la estructura que conserva los conceptos base de la teoría y el modelo (M) es el conjunto modelo teórico que satisface las leyes.

\section{Condiciones de ligadura o "constraints"}

La principal función de las condiciones de ligadura es hacer restricciones en la teoría. Sin embargo, esta característica no solamente es propia de las leyes que influyen en el conjunto de axiomas (impropios) que se han denominado Modelos Potenciales, a no ser que se estén usando modelos por separado, pero no cuando están involucrados dos o más modelos, pues los datos de un modelo pueden ser utilizados en otro. Díez y Moulines dicen lo siguiente: "según la mecánica clásica no puede ser que una partícula $p$ tenga una masa en un modelo $x$ y otra masa diferente en otro modelo $y[\ldots]$ si cierto cohete 
está en el dominio de dos sistemas, uno el sistema Tierra - cohete y otro el sistema Luna - cohete, en ambos modelos ha de tener la misma masa" (Díez y Moulines, 1999, p. 353), obviamente esta situación le hace justicia a la mecánica porque la suma de la masa de las partículas del segundo modelo tiene que ser equivalente a la suma de la masa de las partículas del primer modelo.

\section{T- teoricidad y modelos parciales}

Para los fines de esta exposición recordemos la importancia que tienen estos elementos para la concepción estructuralista, veíamos atrás que según Moulines, dentro de una teoría debemos identificar los $T$-teóricos como los $T$-no-teóricos. Los primeros son aquellos términos de las teorías que son propios de ella, mientras los segundos son algo así como, términos de teorías subsidiarias o anexas.

De esta manera, dentro del conjunto de los modelos potenciales (Mp) distinguimos los elementos teóricos previamente disponibles, denominados dentro del estructuralismo como modelos potenciales parciales (Mpp). Como lo expresa Moulines, "estos modelos se obtienen a partir de los modelos potenciales "recortando" de ellos las entidades $T$ - teóricas". Hasta acá entonces, tenemos la conformación del elemento teórico $\mathrm{K}$, que es como sigue: $\mathrm{K}=<\mathrm{Mp}, \mathrm{Mpp}, \mathrm{M}, \mathrm{GC}>$ (al conjunto de modelos potenciales; al conjunto de los modelos potenciales parciales; a los modelos actuales y a las condiciones de ligadura).

Para comprender el significado de K, adoptemos el ejemplo de Suppes sobre la mecánica newtoniana en el que podemos identificar cada uno de los elementos que lo conforman, a través de la ordenación de tuplos organizados a la manera conjuntista.

$x$ es un sistema de mecánica (newtoniana) de partículas syss def existen $P, T$,

$s, m, f$ tales que: ${ }^{8}$

8 Gráfica tomada del libro de Díez y Moulines "Fundamentos de Filosofía de la Ciencia" "(1) presenta (el número de) los constituyentes de las estructuras. (2) - (6) son los axiomas impropios, meras tipificaciones lógico-matemáticas de las entidades que constituyen la estructura. La idea es que $P$ es un conjunto específico de partículas: en una estructura $x$ determinada ese conjunto contiene sólo la Tierra y la Luna; en otra, el Sol y los planetas; en otra, la Tierra y el péndulo; en otra la Tierra y dos objetos en una polea; etc. $T$ es un conjunto de instantes temporales. $s$ es la función posición, que asigna a cada partícula del sistema un determinado vector-posición en cada instante; en dos veces diferenciable respecto del tiempo, su primera derivada es la velocidad y su segunda derivada es la aceleración. $m$ es la función masa, que asigna a cada partícula un número real positivo, su masa (que es independiente del tiempo). $f$ es 
(1) $x=<P, T, s, m, f>$

(2) $P$ es un conjunto finito no vacío.

(3) $T$ es un intervalo de números reales.

(4) $s$ es una función $P \times T$ en el conjunto de vectores tridimensionales

(tríos ordenados) de números reales, y es dos veces diferenciable sobre $T$.

(5) $m$ es una función de $P$ en el conjunto de numeros reales tal que para todo $p \in P: m(p)>0$.

(6) $f$ es una función de $P \times T \times N$ en el conjunto de vectores tridimensionales (tríos ordenados) de números reales.

(7) Para todo todo $p \in P$ y $t \in T: m(p) . d 2 / d t 2[s(p, t)]=\Sigma i \epsilon N f(p, t, i)$.

De esta manera podemos distinguir dos primeros elementos del elemento teórico K, por un lado están los axiomas impropios que van de (1) a (6) y que son los modelos potenciales (Mp), mantiene los conceptos que soportan la teoría y el modelo; por otro lado tenemos los axiomas propios (7), que es el modelo que cumple la ley.

Los otros dos elementos restantes del elemento teórico $\mathrm{K}$ quedan referenciados como sigue: los modelos potenciales parciales (Mpp) son un subconjunto de los modelos potenciales $(\mathrm{Mp})$, pues dentro de estos últimos debemos diferenciar aquellos modelos que no son propios de la mecánica de Newton. En otras palabras, de $<P, T, s, m, f>$ que conforman los (Mp), "recortamos" los elementos $T$ - teóricos y así obtenemos el subconjunto (Mpp) $<P, T, s>$ que no son genuinos de la mecánica. En cuanto a las condiciones de ligadura, estas se caracterizan por representar "un conjunto de conjuntos de modelos" que se encarga de satisfacer el valor de identidad de masa a pesar de que existan varios modelos que asignen "a cada partícula determinada masa"; dicho de otra manera, los constraints tienen como objetivo el trasladar la información de un modelo hacia otros, sin que se altere el valor de la masa, "si tengo la masa del cohete en el modelo que forma con la Tierra, puedo calcular ciertos valores dinámicos de la Luna gracias a que exporto información sobre la masa del cohete al modelo que forma con la Luna" (op. cit., p. 353). Como se muestra en (7), la masa la puedo interpretar bien sea en el modelo coheteluna o en el modelo cohete-tierra, sin hacer mayores modificaciones, pues

la función fuerza, que asigna a cada partícula en cada instante una serie de vectores-fuerza, las fuerzas actuantes sobre cada partícula en cada instante [...] (7) expresa el segundo principio de Newton: la suma (vectorial) de las fuerzas actuantes sobre una partícula en un instante es igual a la variación de cantidad de movimiento, o como se suele decir, al producto de la masa de la partícula por su vector- aceleración en ese instante" (op. cit., p. 336). 
con las condiciones de ligadura puedo hacer vínculos "intermodélicas" al "transportar la información de un modelo a otro". 9

\section{Aplicaciones intencionales}

La tarea fundamental de las aplicaciones intencionales (representado en el estructuralismo como el conjunto I) es que el núcleo $\mathrm{K}$ anteriormente descrito pueda ser empleado en ciertas realidades empíricas. En el ejemplo al que apelamos atrás de la MCP, pueden ser casos del conjunto de aplicaciones intencionales en esta teoría "el sistema Tierra - Luna, el sistema solar", un péndulo, un perezoso balanceándose en una hamaca o el choque de dos bolas de billar. Aunque es necesario aclarar dos cosas, la primera es que la descripción del conjunto de las aplicaciones intencionales se da a partir de los términos no teóricos, o sea como modelos potenciales parciales. En el caso de la MCP, los I son señalados en función de una partícula ubicada en cierto tiempo y en cierto espacio, pero sin recurrir ni a la fuerza ni a la masa. En otras palabras, el conjunto de las aplicaciones intencionales (I) son un subconjunto de los modelos potenciales parciales (Mpp). En segundo lugar, depende del criterio de los científicos lo que debe contar como el conjunto de las aplicaciones intencionales (I) para poder ejecutar la teoría en sistemas empíricos reales, de ahí que las aplicaciones intencionales posean un fuerte ingrediente pragmático.

\section{La noción de modelo según van Fraassen}

Bas van Fraassen en su tarea de caracterizar su propia concepción sobre la teoría de modelos, presenta más que estructuras conjuntistas, lo que hace más bien es interpretar a los modelos de una teoría como "puntos o trayectorias en un espacio de estados". La pretensión de van Fraassen con esta original noción de lo que puede ser un modelo es que de acuerdo a ciertas variables de un sistema en un momento dado se puede determinar su estado a través de ciertas coordenadas. Por ejemplo, para determinar el estado de un gas debemos

Dice Díez y Moulines: "Debe quedar claro que no hay manera de expresar este tipo de constricciones mediante los axiomas usuales, pues éstos se aplican a modelos sueltos" (op. cit., p. 353). El estructuralismo metateórico plantea la diferencia entre (leyes propias) constricciones a sistemas aislados y que asumen conceptos propios de una teoría; (leyes puente) constricciones a sistemas aislados y que asumen conceptos de teorías distintas; y (condiciones de coherencia o ligadura) "constricciones que se imponen a grupos de sistemas". Sin embargo, para fines expositivos no voy a entrar en detalle sobre la formulación de cada una de estas constricciones, para comprenderlas adecuadamente sugiero ver la última parte del capítulo 10 del libro citado de Díez y Moulines. 
apelar a los valores que lo identifican, tales como el volumen, la presión y la temperatura, así pues, en este caso tendríamos tres magnitudes ordenadas $<v$, $p, t>$ que se refieren a cada componente del gas. Para determinar el estado de un organismo dentro de la genética mendeliana es necesario recurrir a las múltiples características que determinan al fenotipo y a todos los valores que posee el gen para estipular el genotipo, juntas establecen el estado del organismo en cuestión $\langle n f, n g\rangle$, descritas obviamente en una trayectoria de coordenadas. A partir de estos dos ejemplos podemos ver que "los estados se identifican por tanto en general con puntos en un determinado sistema de coordenadas, de tantas dimensiones posibles como componentes tengan los estados" (Diez, Moulines, 1999, p. 342). En el caso del estado del gas le corresponde un espacio tridimensional (volumen, presión y temperatura) $\mathrm{y}$ en el caso del organismo le corresponde un espacio bidimensional ( $n$ características del fenotipo y $n$ valores del genotipo). Las posibles $n$ secuencias que determinan la dimensión de un estado son los puntos o trayectorias que hay en él.

Ahora, las leyes de una teoría también juegan un papel importante a la hora de establecer el espacio de estados, pues estas tienen la labor de hacer restricciones, por lo menos en dos sentidos: puede que las leyes fijen ciertas trayectorias o que impongan ciertas exclusiones a algunas regiones del espacio fase. Esto es así porque las leyes establecen un subconjunto del espacio de estado que se refiere a los elementos lógicamente o nómicamente posibles, que de antemano la teoría ha prefijado.

Ahora, parte de la definición del instrumentalismo de van Fraassen es que las teorías cientificas son instrumentos que modelan, por lo menos, una parcela del mundo y que en este enfoque es necesario tener presente las siguientes implicaciones:

a) Las teorías científicas son simplemente instrumentos útiles que se relacionan con una porción del mundo.

b) Las teorías científicas más que ser verdaderas son empíricamente adecuadas.

c) En cuanto a los entes inobservables implicados por la teoría se debe, según los instrumentalistas, suspender el juicio. No hay compromiso con la verdad.

La ventaja de este punto de vista es que: 1) no nos compromete a aceptar que las teorías representan de una manera fiel y exacta como es el mundo, las teorías son solo idealizaciones; y 2) pensar que las teorías son un conjunto de enunciados implica que si una o varias proposiciones que forman parte de una teoría fueran falsas, se sigue que la teoría en su conjunto sería también falsa. 
Las teorías son empíricamente adecuadas cuando describen efectivamente los fenómenos que pertenecen a un campo determinado. Van Fraassen lo expresa de la siguiente manera, "la teoría es empíricamente adecuada si tiene algún modelo tal que todas las apariencias son isomórficas con las subestructuras empíricas de ese modelo" (van Fraassen, 1996, p. 89).

Recordemos precisamente que la física newtoniana tiene como verdadera la existencia del "espacio absoluto" y del "éter", sin embargo las objeciones de Leibniz y posteriormente de Mach y Einstein apuntaban a que la idea de espacio vacío tiene sentido desde una perspectiva relacional, pero no es una entidad abstracta que haga parte del mobiliario del mundo como lo entendía Newton. Pese a esto, no es correcto considerar que la mecánica newtoniana este equivocada en su totalidad.

Es importante detenernos en dos aspectos de esta postura antirrealista que asume van Fraassen, las teorías cuya función es salvar los fenómenos y las teorías como instrumentos de cálculo.

Para el instrumentalismo las teorías son construcciones que tienen la finalidad de explicar y describir solamente lo que es empíricamente observable; o más bien lo que en apariencia le corresponde al mundo. ${ }^{10} \mathrm{En}$ términos generales, en esto consiste la labor de salvar los fenómenos; además, las teorías son instrumentos de cálculo en el sentido de que sirven para realizar predicciones en el dominio de los observables. Andrés Rivadulla (2007) expone estos dos atributos del instrumentalismo de la siguiente manera:

Los modelos teóricos constituyen constructos tendentes a 'salvar los fenómenos' y a facilitar predicciones empíricamente contrastables acerca de los dominios empíricos de su competencia...Los modelos teóricos no contienen conocimiento del Mundo, sino que constituyen meras herramientas para manejarnos predictivamente con la naturaleza... (Rivadulla, 2007, pp. 16-17)

10 En el caso de van Fraassen, su propuesta del Empirismo Constructivo consiste en que la actividad científica depende de la construcción de modelos que se deben "adecuar" a ciertas parcelas del mundo. Esto rompe con el empirismo clásico en el sentido en que en éste el conocimiento parte de la experiencia y debe corresponderse (bis a bis) con el mundo. Así, la ciencia es "construcción de modelos que deben ser adecuados a los fenómenos, y no descubrimiento de la verdad respecto de lo inobservable" (van Fraassen, 1996, p. 20). Ronald Giere (2005 y 1992) por su parte, quien también se adhiere al enfoque semántico de las teorías, propone una teoría alternativa a la de van Fraassen, la denomina Realismo Constructivo en el sentido en que si las teorías son exitosas es porque dicen tal cual es el mundo y comparte con van Fraassen el asunto de que los modelos teóricos son construcciones de los mismos científicos. En oposición a la "adecuación empírica" de van Fraassen la noción de "similitud" o "semejanza" es lo que presenta Giere. Dice: "Una hipótesis teórica afirma la existencia de semejanza entre un cierto modelo teórico y un sistema real designado" (Giere, 1992, pp. 117-118). Sin embargo, el debate entre el empirismo constructivo y el realismo constructivo no es tanto con el asunto del enfoque semántico de las teorías, la discusión interesante es sobre la controversia entre el realismo y el antirrealismo en la filosofía de la ciencia, por tal razón no creo pertinente exponer en este artículo las diferencias en detalle de ambos autores. 
De acuerdo con este panorama, una de las implicaciones más fuertes de estos dos aspectos del instrumentalismo es que la verdad de las teorías queda en un segundo lugar, si es que le corresponde alguno, lo único que les incumbe es si las teorías como instrumentos son útiles o no para explicar tanto los fenómenos observables como para realizar predicciones con relación a ellos. Tal vez sea el mismo van Fraassen quien ofrece una diferencia más aguda entre las dos actitudes epistemológicas que han influenciado a la filosofía de la ciencia, entre los que asumen el correspondentismo de la concepción heredada y los que defienden el enfoque semántico de las teorías, "la afirmación de adecuación empírica es bastante más débil que la afirmación de verdad, y la resistencia a aceptar nos libera de la metafísica" (van Fraassen, 1996, p. 94).

\section{A modo de conclusión}

Si bien en el presente artículo he intentado mostrar la distancia que han tomado dentro de la concepción semanticista de las teorías, tanto el estructuralismo como el empirismo constructivo en la manera de comprender la naturaleza de las teorías científicas, es importante señalar que otra de las diferencias epistemológicas es que van Fraassen no ha tomado una distancia clara con la clásica taxonomía entre observacional/no-observacional, pues toda relación isomórfica entre la teoría o partes de la teoría (subestructuras empíricas de los modelos teóricos) es con los fenómenos (observables), en vez de las aplicaciones intencionales o modelos de datos.

Sin embargo, una de los inconvenientes que van Fraassen advierte en el enfoque estructuralista es que es innecesario hacer toda la labor de reconstrucción de teorías, para él es suficiente con la formalización general en "espacios de fase" porque esta propuesta le hace justicia a la manera en la que operan los científicos. Esto es, los científicos no toman en cuenta las reconstrucciones en el laboratorio, a pesar de que es importante reconocer que los estructuralistas son los que hacen un análisis más fino y minucioso de las teorías.

Con todo, considero que la manera de concebir las teorías científicas dentro de la familia semanticista, o por lo menos en las dos versiones que aquí presento, muestra que la noción de modelo (en el sentido matemático) es muy interesante desde el punto de vista filosófico, entre otras cosas porque podemos prescindir de la concepción tan arraigada sobre la verdad (por correspondencia) y poder tener varios matices para juzga el éxito una teoría en particular, como su adecuación empírica o su utilidad práctica. Justamente, si la afinidad más notoria dentro de la familia semanticista radica en que interpretar 
a las teorías sintácticamente es ineficaz para comprender su naturaleza y de ahí la aceptación del concepto de modelo, la diferencia más puntual está en la manera sobre cómo concebirlo. Es decir, como ocurre en muchas familias, los lazos se rompen hasta por el parentesco que los juntó.

\section{Bibliografía}

ADAMS, E. "The Foundations of Rigid Body Mechanics and the Derivation of Its Laws from Those of Particle Mechanics". In: L. Henkin, P. Suppes, A. Tarski (ed.). The Axiomatic Method (pp. 250-265). Amsterdam: North Holland, 1959.

BALZER, W., MOULINES C., SNEED J. "An Architectonic for Science. The Structuralist Program”. Dordrecht: Reidel, 1987.

CARNAP, R. "Meaning and Necessity. A study in semantics and modal logic". Chicago: The University of Chicago Press, 1956.

CASANUEVA, M. "Los Modelos en la Filosofía de la Ciencia del Siglo XX". In: A. L. Austín. El Modelo en la Ciencia y la Cultura. México: Editorial Siglo XXI UNAM, 2005.

DÍEZ, J., MOULINES, C. U. "Fundamentos de Filosofía de la Ciencia". Barcelona: Ariel, 1999.

GIERE, R. "La Explicación de la ciencia. Un Acercamiento Cognoscitivo". México: Consejo Nacional de Ciencia y Tecnología, 1992.

149-165, 2005.

. "Scientific Realism: Old and New Problems". Erkenntnis, Nr. 63, pp.

HEMPEL, C. "Filosofía de la Ciencia Natural". Madrid: Alianza Editorial, 1980.

MOULINES, C. U. "Exploraciones metacientíficas". Madrid: Alianza Editorial, 1982. . "La Concepción Estructuralista de la Ciencia". Discusiones Filosóficas,

Nr. 5-6, pp. 11-21, ene.-dic. 2002.

RIVADULLA, A. "Restricciones sin refutaciones de dominios de teorías físicas. Elementos para el debate realismo - instrumentalismo". Eidos, Nr. 6, pp. 10-25, 2007. SNEED, J. "The Logical Structure of Mathematical Physics". Dordrecht: Reidel, 1971.

SUPPE, F. "La Estructura de las Teorías Científicas". Madrid: Editora Nacional, 1979. SUPPES, P. "Estudios de Filosofía Metodología de la ciencia”. Madrid: Alianza, 1988. TARSKI, A. "Logic, semantics, metamathematics". Oxford: Clarendon Press, 1956. VAN FRAASSEN, B. "Laws and Symetry". Oxford: Clarendon Press, 1989. . "La imagen científica". Barcelona: Paidós, 1996. 